\title{
Capture Process by Shallow Donors in Silicon at Low Temperatures
}

\author{
A. Palma, J.A. Jiménez-Tejada, A. Godoy and P. Cartujo \\ Departamento de Electrónica y Tecnología de Computadores, Facultad de Ciencias, Universidad de \\ Granada, 18071 Granada, Spain
}

\begin{abstract}
Thermal capture by shallow attractive impurities has been studied by the Monte Carlo method at low temperatures. In order to model this process, the perturbation produced by the highest excited impurity states associated to the ideal Coulombic potential and the multiphonon mechanism were combined in the same simulation procedure. These excited states are considered to produce an energy quasicontinuum or impurity band where the carrier can move assisted by lattice phonons. To characterize the ground-state capture, the transition rates of multiphonon transitions from the impurity band and from the conduction band have been included. Electron capture cross sections have been calculated as a function of the temperature and compared compared with experimental data from the $\mathrm{P}^{+}, \mathrm{As}^{+}$and $\mathrm{Sb}^{+}$shallow donors in $\mathrm{Si}$, achieving a good fitting with the Huang-Rhys factor, S, as the only free parameter.
\end{abstract}

\section{INTRODUCTION}

The most powerful numerical methods are now being used to revise old hypotheses and models on the microscopic interaction between carriers and impurities related to generation-recombination processes. In this area, some of the most important mechanisms in thermal capture studied have been the multiphonon emission [1] and the cascade process [2]. These works demonstrated the importance of appropriate modelling of the different elements involved in the capture process, such as bound and free states, and the perturbation produced by impurities.

In this work, we have studied capture by a type of defects which can not be interpreted at any temperature by any single capture mechanism mentioned above: capture by shallow levels. Multiphonon emission is shown to be necessary even for shallow centers due to the separation between the deepest excited levels associated to these centers. The capture cross section is one of the magnitudes most affected by this interaction: strong thermal dependence with temperature is observed experimentally. Precisely in order to explain this thermal behavior of the cross section, the so-called two stage model was proposed [3-5]. Their analytical treatments supposed a simplified scheme of this kind of trap capture, where the influence of the excited states were reduced to the interaction with a single effective level and some parameters were required to fit experimental data. The theoretical background of the two-stage capture model is very useful; however, other aspects not taken into account due to their complexity can be added to this model in order to obtain a more physical view of the phenomenon. In fact, we will show how, by using the Monte Carlo method, most of the simplifications made in previous models to achieve an analytical result can be set aside. Our numerical procedure is based on the combination in a single simulation of two capture mechanisms: the cascade process and multiphonon emission, including the highest excited levels as an energy quasicontinuum. The calculation of capture cross sections of this kind of centers will be used as a way of testing our model. 


\section{CAPTURE MODEL BY SHALLOW IMPURITIES}

The potential created by an ionized shallow impurity in a lattice is known to produce a set of excited states next to the band edge. These excited states favor the capture of carriers when the temperature and the applied electric field permit carrier distribution close to the band edge, whereas their influence becomes unimportant for carriers far from this edge.

A schematized representation of the perturbation created by an attractive trap and the capture transitions induced by it is shown in Fig. 1. The cascade process (one-lattice phonon-assisted transitions), named (1) in Fig.1, has been selfconsistently introduced using the Monte Carlo method. We have numerically implemented a Coulombic perturbation produced by the impurity by modifying of the lattice-scattering probabilities and allowing the carrier to move across the excited states as an energy continuum. In order for the carrier to reach ground state, multiphonon probabilities are proposed and introduced in the "perturbed" situation given above. The transition rates to ground level will be calculated differently depending on where the carrier comes from: an excited impurity state (transition (2) in Fig. 1) or a band level (transition (3) in Fig.1). A detailed explanation of our numerical procedure is given below.

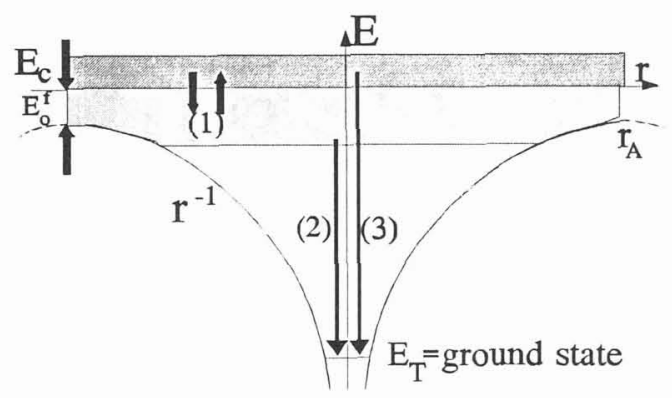

Figure 1. Schematic plot of the total space energy of the conduction band of Si perturbed by the potential created by a Coulombic center. Ground state $\mathrm{E}_{\mathrm{T}}$ and the impurity band created by the highest excited levels are represented. The energy $E_{0}{ }^{f}$ corresponding to the energy limit of the center influence in a randomly distributed concentration is also plotted. The generation-recombination processes in the excited levels (transition (1)), the capture from the impurity band (2), and the direct transition from the conduction band (3) are taken into account.

The cascade process for electrons was numerically implemented by considering how the Coulombic perturbation, due to the attractive potential created by the impurity, affects the state density, the scattering probabilities, and the phonon energies [6]. As mentioned, we have assumed that the highest excited levels behave like a quasicontinuum, or an impurity band. We have taken the first excited state of the Coulombic potential in the semiconductor, $\mathrm{E}^{1}$, as the minimum of this quasicontinuum, $\mathrm{E}_{\mathrm{c}}-\mathrm{E}^{\mathrm{l}}=-11.5 \mathrm{meV}$. Although the impurity band width may be different for each trap, we will show below that, according to this model, the transition to ground state occurs from the highest excited states of this band, with the importance of the lowest levels being negligible.

Let $E$ be the total carrier energy and $E_{k}$ its kinetic energy. To include the perturbation caused by the impurity potential, the phonon-scattering rates, $\mathrm{P}(\mathrm{E})$, are then spatially averaged to an impurity volume introducing the dimensionless distance $x=r / r_{A}$ :

$$
\left\langle P\left(E_{k}\right)>=3 \int_{0}^{x_{m}} P\left(E+\frac{E_{o}^{f}}{x}\right) x^{2} d x,\right.
$$

where $r$ is the distance related to impurity, and $r_{A}=\left(N_{D}{ }^{+}\right)^{-1 / 3}$ is taken as the average semi-distance between impurities in a sample with an impurity concentration of $N_{D}{ }^{+}$. If $E>-E_{o}^{f}, x_{m}=1$, and if $-E^{1}<E<-E_{o}^{f}$, then $x_{m}=-E_{o}^{f} / E$, where $E_{o}^{f}$ is the potential energy in $r=r_{A}$, and $E_{o}^{f}=e^{2} / 4 \pi \varepsilon r_{A}$, with $\varepsilon$ being the semiconductor dielectric constant and e the electron-charge magnitude. Zero-energy is located at the band edge, while 
the impurity states have negative energy values and the free states have positive ones. Assuming that all the relative positions to the center have the same probability, we define an effective energy, $\varepsilon_{\mathrm{m}}$, that plays the same role as the carrier energy in an unperturbed lattice but that takes into account the effects of the Coulombic potential. Equation 1 can in consequence be written as follows:

$$
3 \int_{0}^{x_{m}} P\left(E+\frac{E_{o}^{f}}{x}\right) x^{2} d x=3 P\left(\epsilon_{m}\right) \int_{0}^{x_{m}} x^{2} d x=P\left(\epsilon_{m}\right) x_{m}^{3} .
$$

For phonon-scattering mechanisms, the numerically calculated $\varepsilon_{\mathrm{m}}$ values were:

$$
\epsilon_{m}=\left\{\begin{array}{l}
E+\frac{4}{3} E_{o}^{f}, \quad E>-E_{o}^{f} \\
\frac{|E|}{3}, \quad-E^{1}<E<-E_{o}^{f} .
\end{array}\right.
$$

Therefore, from Eqs. 2 and 3, the scattering probabilities were calculated as

$$
P(E)=\left\{\begin{array}{lc}
P\left(E+\frac{4}{3} E_{o}^{f}\right), & E>-E_{o}^{f} \\
{\left[-\frac{E_{o}^{f}}{E}\right]^{3} P\left(\frac{|E|}{3}\right),} & -E^{1}<E<-E_{o}^{f} .
\end{array}\right.
$$

Within this model, the carrier is allowed to move through band and impurity band states according to the probabilities given in Eq. 4. More details about this procedure are given in Ref. 6.

Once excited impurity states and their interactions with the band have been modelled, the multiphonon transition rate to ground level, $\mathrm{E}_{\mathrm{T}}$, must be calculated. According to Ridley's approach for low temperatures and weak coupling, the capture probability can be written by [1]

$$
W^{m p h}=W_{0}(n+1)^{p} e^{-2 n S}, \quad p^{2}>4 S^{2} n(n+1),
$$

where for $S<<p$

$$
W_{o}=\frac{\omega \pi}{(\hbar \omega)^{2}} \frac{p S^{p-1} e^{-S}}{p !}\left[0.26|V|^{2}+0.18|V \cdot \Delta|^{2} \frac{p-1}{S}\right], \quad n=\left(e^{\frac{\hbar \omega}{K_{B} T}}-1\right)^{-1}
$$

$K_{B}$ is the Boltzmann constant, $\hbar \omega$ the phonon energy, $p=\left(E_{\mathrm{T}}+\mathrm{E}\right) / \hbar \omega$ the number of emitted phonons, and $\mathrm{S},|\mathbf{V}|^{2}$, and $|\mathbf{V}-\Delta|^{2}$ are three matrix elements that represent the Huang-Rhys factor and the interactions between the final and initial states via the electron-phonon potential:

$$
\left.S=|\Delta|^{2}, \quad \Delta \approx-\frac{1}{2^{1 / 2} \hbar \omega}<b|\boldsymbol{U}| b\right\rangle, \quad V=\langle f|\boldsymbol{U}| b\rangle
$$

where $b$ and $f$ are the bound and free states respectively, and the electron-phonon coupling is:

$$
H_{e p}=-\boldsymbol{U} \boldsymbol{Q} \text {. }
$$

$\mathbf{U}$ and $\mathbf{Q}$ are $\mathbf{N}$-dimensional vectors whose components are associated with the phonon modes ( $\mathrm{N}$ is the number of phonon modes). $\mathbf{Q}$ is the vector of normal coordinates and $\mathbf{U}$ the potential for the electronphonon coupling.

To calculate the probability given in Eq. 5, some models of these magnitudes have been assumed. The following approximate models were considered: 
i) For the electron-phonon coupling, we have considered the optical deformation-potential

$$
\boldsymbol{U}=D(\boldsymbol{q}) e^{i q \cdot \boldsymbol{r}}
$$

where $|D(q)|^{2}=A_{D} q^{0}, q$ is the phonon wave-vector, and $A_{D}$ is a material constant. Although the phonon energy involved in the multiphonon transition is unknown, we chose lattice phonons in order to fix this parameter in probability. We have taken the TA phonon, $\hbar \omega=12 \mathrm{meV}$, as the phonon emitted in transitions to shallow impurity levels in $\mathrm{Si}$.

ii) An acceptable approximation for the bound ground state in the impurity is the s-orbital wavefunction:

$$
\langle b|=\frac{A}{r} e^{-\frac{r}{a_{H}}}
$$

where $a_{H}$ is the energy level of a hydrogen-like center, $a_{H}{ }^{*}=\left(\hbar^{2} / m^{*}\right) /\left(e^{2} / 4 \pi \varepsilon\right)$ with $m^{*}$ the carrier effective mass.

iii) For the free carrier state close to the impurity, it is necessary to distinguish between a direct transition from the band or from an excited level. In the direct transition, in accordance with many previous calculations, we have taken

$$
\langle f|=\left(\frac{C_{o}}{V}\right)^{\frac{1}{2}}, \quad C_{o}=-\frac{2 \pi \eta}{e^{2 \pi \eta}-1}, \quad \eta=-\left(\frac{R_{H}}{E-E^{1}}\right)^{1 / 2}
$$

$C_{o}$ is the Coulomb factor [7], $E$ is the carrier energy, and $V$ is the crystal volume. When the carrier is captured by the ground level from an excited state, a new initial state volume, $\mathrm{V}_{\mathrm{ex}}$, must be considered. For a Coulombic potential, the radius should be given by the Columbic orbit radius;

$$
V_{e x} \propto\left(\frac{e^{2}}{4 \pi \epsilon|E|}\right)^{3}, \quad-E^{1}<E<-E_{o}^{f} .
$$

With all these models and using Stirling's formula, the matrix elements can be calculated. Thus, the multiphonon capture probability of the ground state can be approximated by

$$
W^{m p h} \propto \frac{288 \pi^{2} \omega a_{H}^{*^{3}} C_{o}}{(2 \pi p)^{1 / 2}(p / e)^{p}}\left\{\begin{array}{l}
V^{-1} \\
V_{e x}^{-1}
\end{array}\right\} p S^{p} e^{-S}[0.26+0.18(p-1)](n+1)^{p} e^{-2 n S}
$$

\section{NUMERICAL TREATMENT}

This attractive impurity model has been implemented in the simulation of electron transport in the silicon conduction band by the standard Monte Carlo method. The task of incorporating the multiphonon probability in a Monte Carlo simulation was solved by restricting the motion of the carrier to one impurity space [8]. Therefore, in a sample with an ionized impurity concentration of $\mathrm{N}_{\mathrm{D}}{ }^{+}$, the volume occupied by a band state can be approximated by $\mathrm{V}=\left(\mathrm{N}_{\mathrm{D}}{ }^{+}\right)^{-1}$. Thus, the real multiphonon probabilities included were

$$
<W^{m p h}>\approx \frac{288 \pi^{2} \omega a_{H}^{*^{3}} C_{o}}{(2 \pi p)^{1 / 2}(p / e)^{p}}\left\{\begin{array}{c}
N_{D}^{+} \\
V_{e x}^{-1}
\end{array}\right\} p S^{P} e^{-S}[0.26+0.18(p-1)](n+1)^{p} e^{-2 n S}
$$

using the upper factor for energies greater than $-E_{o}{ }_{0}$ and the lower one for $-E^{1}<E<-E_{0}{ }^{f}$. These capture 
rates were incorporated into the perturbed conduction band plotted in Fig. 1.

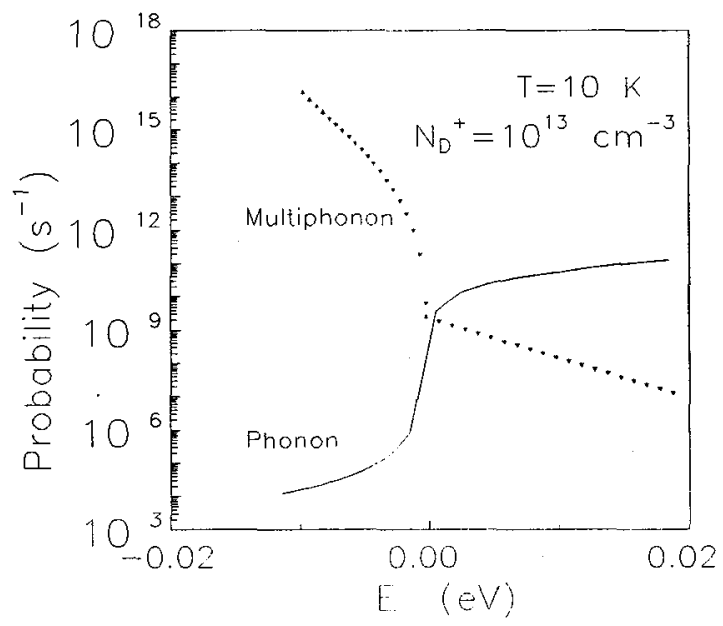

Figure 2. Probabilities of phonon-scattering (dashed line) and multiphonon emission (solid line) as a function of the carrier energy. The calculation was carried out for a shallow attractive donor with the ground state located at $0.044 \mathrm{eV}$ below the conduction band of $\mathrm{Si}$ at $10 \mathrm{~K}$.

In Fig. 2 the probability given by Eq. 14 has been plotted as a function of the carrier energy, E (solid line), together with the sum of the phonon-scattering mechanisms (dashed line). The plot has been carried out for the shallow level at $0.044 \mathrm{eV}$ below the conduction band edge, with a Huang-Rhys factor of $S=0.25$ and an ionized donor concentration of $N_{D}{ }^{+}=10^{13} \mathrm{~cm}^{-3}$ at $10 \mathrm{~K}$. In this figure we can observe that one electron has a non-negligible phonon-scattering probability in the excited states (negative energies). The electron can move across these impurity states and the conduction band according to the probabilities given in Eq. 4. Re-emission processes are taken into account from the highest excited levels to the band till a multiphonon transition to the ground state occurs. The multiphonon capture probability is not negligible in the conduction band, and therefore, the direct capture from any impurity state and band energy level is allowed. As we can also see, the minimum of the defined impurity band can reasonably be taken when the multiphonon probability dominates over the rest of the rates, since for these lower energies the capture will be very fast. For the analyzed centers this happens at a few milielectronvolts below the minimum of the conduction band.

Given the probability dependencies with the energy shown in Fig. 2, at low temperatures the carrier tends to go down into the excited states, thus favoring a fast capture. When the temperature goes up, the electron gains thermal energy and has a lower probability of being close to the band edge. In addition, the remission rate from the negative levels increases, and therefore the capture cross section will strongly decrease. All these behaviors will be reflected in the simulation results, as we will show.

The numerical procedure was as follows: one electron is introduced with the thermal energy corresponding to the lattice temperature and is allowed to move without considering the multiphonon and cascade process in order to avoid dependencies with the initial carrier state. After a certain number of scattering events, the Coulombic perturbation and multiphonon probability are introduced. At precisely the moment when the multiphonon mechanism is stochastically chosen, the electron is considered captured, and the mean velocity and time elapsed that the electron spends in the conduction band, since the capture-process inclusion, the so-called capture time, are recorded. This one-electron procedure is repeated for a very large number of carriers, and the average values of the velocities, $\langle\mathrm{v}\rangle$, and the capture times, $\left\langle\tau_{\mathrm{c}}\right\rangle$, are used to calculate the average thermal capture cross section for electrons, $\sigma_{\mathrm{n}}$, $\left.\sigma_{\mathrm{n}}=\left(\mathrm{N}_{\mathrm{D}}{ }^{+}<\mathrm{v}\right\rangle\left\langle\tau_{\mathrm{c}}\right\rangle\right)^{-1}$. 


\section{COMPARISON WITH EXPERIMENTAL DATA AND CONCLUSIONS}

The numerical capture cross sections calculated by the Monte Carlo method and experimental measurements $[9,10]$ are compared and good fittings are achieved for the $\mathrm{P}^{+}, \mathrm{As}^{+}$and $\mathrm{Sb}^{+}$shallow levels in Si. Since the Huang-Rhys factor, S, was unknown, it was used to fit both curves and it remained as the only free parameter of the simulation. Concretely, we have obtained $S=0.25$, value according to low coupling for these type of impurities.

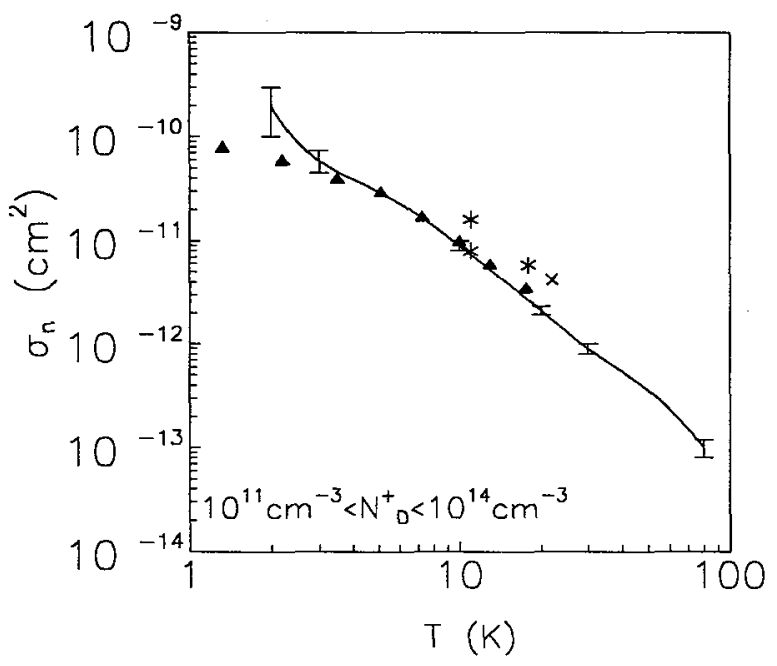

Figure 3. Experimental $[9,10]$ (symbols) and numerical (line with error bars) thermal dependence of the capture cross section for shallow donors in $\mathrm{Si}\left(\mathrm{P}^{+}\right.$, triangles; $\mathrm{As}^{+}$, asterisks and $\mathrm{Sb}^{+}$, crosses). A value of $\mathrm{S}=0.25$ was used to obtain the fitting.

In summary, a capture model by ideal Coulombic-attractive levels has been developed and numerically implemented by the Monte Carlo method. The method is based on the combination of cascade and multiphonon processes to obtain a more realistic model of capture by this kind of traps. This model has been tested by numerical calculation of the thermal capture cross section of shallow donors in Si. The strong thermal dependence of this magnitude has been satisfactorily reproduced with a single free parameter in the whole model: the Huang-Rhys factor, $S$.

\section{References}

[1] Ridley B.K., Solid-State Electron. 21, (1978) 1319-1323.

[2] Lax M., Phys. Rev. 119, (1960) 1502-1523.

[3]Gibb R.M., Rees G.J., Thomas B.W., Wilson L.H., Hamilton B., Wight D.R. y Mott N.F., Phylos. Mag. 36, (1977) 1021-1034.

[4] Landsberg P.T. and Dhariwal S.R., Phys. Rev. B 39, (1989) 91-93.

[5] Dhariwal S.R. and Landsberg P.T., J. Phys.:Condens. Matter 1, (1989) 569-584.

[6] Palma A., Jiménez-Tejada J.A., Godoy A., López-Villanueva J.A. and Carceller J.E., Phys. Rev. B 51, (1995) 14147-14151.

[7] Amato M.A. and Ridley B.K., J. Phys. C 13, (1980) 2027-2039.

[8] Reggiani L., Varani L., Mitin V. and VanVliet C.M., Phys. Rev. Lett. 63 (1989) 1094-1097.

[9] Norton P. and Levinstein H., Phys. Rev. B 6 (1972) 489-497.

[10] Abakumov V.N., Perel' V.I. and Yassievich I.N., Sov. Phys. Semicond. 12 (1978) 1-18. 